\title{
Determination of the quality of results obtained by various numerical methods for BSD
}

\author{
Artur Krzyżak, Piotr Łukasik, Krzysztof Janc \\ AGH University of Science and Technology, Faculty of Geology, \\ Geophysics and Environmental Protection, \\ A. Mickiewicza 30, 30-059 Kraków, Poland \\ Email: akrzyzak@agh.edu.pl,pioluk@student.agh.edu.pl,krzysztof.janc@gmail.com
}

\begin{abstract}
This article describes the determination of the quality of results obtained by various numerical methods for BSD (B-matrix Spatial Distribution). In order to verify the influence of the numerical error on the real data, two datasets acquired using two types of phantoms (isotropic and anisotropic) and the reference random data were analyzed. Additionally examined aspect was the duration of the calculations. The research were carried out on six of numerical methods for solving systems of equations (Gauss, Gauss Jordan, LU, Gauss with partial pivoting, LU (numerical recipes), Gauss-Jordan (numerical recipes)).
\end{abstract}

\section{INTRODUCTION}

$\mathrm{D}$ IFFUSION Tensor Imaging (DTI) is one the of the most widely used methods of imaging the anisotropic biological structures such as white and grey matter andskeletal muscles. DTI is an imaging technique basing on the phenomenon of nuclear magnetic resonance (NMR), which allows for measurement of diffusion coefficient and its direction, expressed in the form of diffusion tensor. Scientific reports present a broad range of clinical applications of the described method e.g. nerve fibers (tractography), diagnosis of cerebral ischemia, multiple sclerosis, epilepsy, metabolic disorders, tumors of the brain [1-3], and studies on the brain function [4].

Diffusion tensor is a symmetrical $3 \times 3$ matrix containing six independent elements. To derive all elements of the diffusion tensor one reference and 6 diffusion weighted images (obtained with various non collinear orientations of a diffusion gradient vector) are required. The relations between the signal and diffusion tensor is described by Stejskal-Tanner equation [6]:

$$
\ln \left(\frac{S_{n}}{S_{0}}\right)=-\mathbf{b}: \mathbf{D}=-\sum_{i, j=1}^{3} b_{i j} D_{i j}
$$

where $S_{n}(b)$ and $S\left(b_{0}\right)$ are the signal intensities with and without nth diffusion sensitizing gradient, respectively; $b_{i j}$ is a component of the diffusion gradient b matrix; $D_{i j}$ is a component of the diffusion tensor $D$. The colon designates the generalized dot product. Each direction of a diffusion sensitizing gradient is described by individual matrix [5].

Computations on floating-point numbers are biased with an error resulting from numerical representation of the numbers. Only a finite length string of binary words can be used for representation of a number, what in the case of irrational values (i.e. with infinite binary expansion) such as $\pi$ or Euler's number, leads to a necessary rounding and loss of the precision.

Another type of an error is the cut-off error. It occurs during computing as a result of decreasing the number of operations, e.g. during computing an infinite Taylor series while calculating the value of $\ln$, extremely important fact is that a minor numerical error can grow during further calculations (e.g. multiplication of small values by larger ones) and cause a major error in the result. The aim of this work is to test the influence of the numerical error on the result of the DTI method. The errors stemming from the fact that the measurements of physical quantities can be done only with the limited accuracy are neglected here.

Several numerical methods for calculating a system of linear equations such as the method of Gauss elimination and its variations (Gauss-Jordan, Gauss with partial pivoting), LU method and Cramer's rule, were implemented. Additionally the implementations of Gauss elimination method and LU decomposition from the Numerical Recipes were added. The following experimental equipment specification were used: Processor Intel Core Intel Core i7-4700MQ@ 2.4 GHz, 8 GB RAM.

\section{VERIFICATION OF THE METHODS}

The first stage of the work was checking the correctness of the implemented methods on random data. For this purpose, each independent value of bn matrix and $\mathrm{D}$ tensor were drawn from the range -1 to 1 . By transforming the equation (1) one obtains the formula expressing $\mathrm{Sn}$ value:

$$
S_{n}=\mathrm{e}^{-\mathrm{b}_{\mathrm{n}}: \mathbf{D}} S_{0}
$$


For final determination of $S_{n}$ one needs a value of $S_{0}$ which is also drawn from the range $[-1 ; 1]$.

Equation (1) has the following form in the matrix representation:

$\left[\begin{array}{cccccc}-b_{x x 1} & -b_{y y 1} & -b_{z z 1} & -2 b_{x y 1} & -2 b_{x z 1} & -2 b_{y z 1} \\ -b_{x x 2} & -b_{y y 2} & -b_{z z 2} & -2 b_{x y 2} & -2 b_{x z 2} & -2 b_{y z 2} \\ -b_{x x 3} & -b_{y y 3} & -b_{z z 3} & -2 b_{x y 3} & -2 b_{x z 3} & -2 b_{y z 3} \\ -b_{x x 4} & -b_{y y 4} & -b_{z z 4} & -2 b_{x y 4} & -b 2_{x z 4} & -2 b_{y z 4} \\ -b_{x x 5} & -b_{y y 5} & -b_{z z 5} & -2 b_{x y 5} & -2 b_{x z 5} & -2 b_{y z 5} \\ -b_{x x 6} & -b_{y y 6} & -b_{z z 6} & -2 b_{x y 6} & -2 b_{x z 6} & -2 b_{y z 6}\end{array}\right]\left[\begin{array}{c}D_{x x} \\ D_{y y} \\ D_{z z} \\ D_{x y} \\ D_{x z} \\ D_{y z}\end{array}\right]=\left[\begin{array}{l}\ln \left(S_{1} / S_{0}\right) \\ \ln \left(S_{2} / S_{0}\right) \\ \ln \left(S_{3} / S_{0}\right) \\ \ln \left(S_{4} / S_{0}\right) \\ \ln \left(S_{5} / S_{0}\right) \\ \ln \left(S_{6} / S_{0}\right)\end{array}\right]$

(3)

In the case of the presented test, all values in the above equation are known. In DTI, the problem lies in determination of unknown values of the diffusion tensor $\mathrm{D}$. To do that, a system of equations containing six unknown variables has to be solved. By solving the system of equations for randomly generated $b$ and $S_{n}(3)$, new values of diffusion tensor $\mathrm{D}^{\prime}$ were determined. Comparing the values of initially determined tensor $\mathrm{D}$ with calculated $\mathrm{D}^{\prime}$ one obtains the numerical error $\varepsilon$.

$$
\varepsilon=\sum_{i, j=1}^{3}\left|D_{i j}-D^{\prime}{ }_{i j}\right|
$$

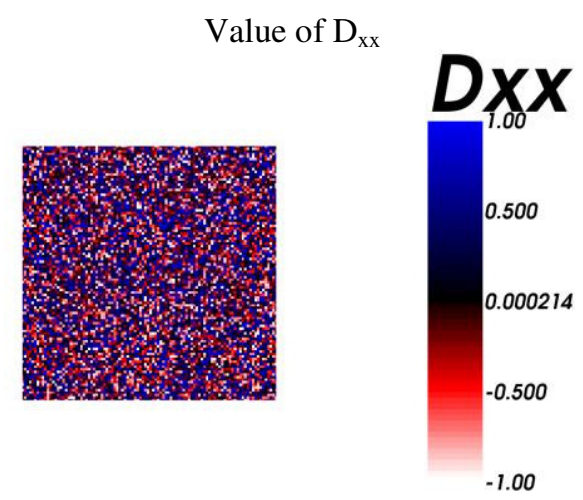

Distribution of the error $\varepsilon$

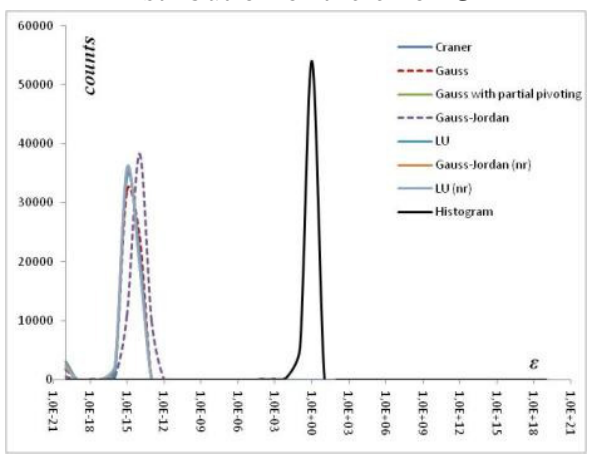

Fig. 1 Distribution of the numerical error $\varepsilon$ for all elements of tensor D and value of the $\mathrm{xx}$ element of randomly generated diffusion tensor.

On the basis of Fig. 1 one can conclude that the numerical error independently from the method chosen, is 15 orders of magnitude smaller than values of components of the drawn
Tensor D, what confirms correctness of the implementation of the methods.

\section{COMPARISON OF THE METHODS}

In the case of DTI data, the exact value of tensor D is unknown, what makes the direct determination of the numerical error impossible. However, the error can be estimated alternatively. In DTI one measures values of $S_{n}$ for the assumed gradient $b_{n}$. Solving the system of equations (3) for $S_{n}$ and $b_{n}$ data the values of Tensor $D$ (with a certain numerical error) are obtained. Then, using the formula (2) $S_{n}^{\prime}$ values were determined. Comparing the input values $S_{n}$ with re-calculated $\mathrm{S}_{\mathrm{n}}^{\prime}$, one obtains indirect numerical error $\xi$ :

$$
\xi=\sum_{n=1}^{6}\left|S_{n}-S_{n}^{\prime}\right|
$$

In order to verify the influence of the numerical error on the real data, two datasets acquired using two types of phantoms (isotropic and anisotropic) and the reference random data were analyzed. It is important that real data belong to distributions which are far from the uniform distribution $(-1.0,1.0)$.

On the basis of Fig. 2 it can be stated that, for the randomly generated data, the indirect error for all numerical methods is 15 orders of magnitude smaller than the average value of the input data. But on the other hand, in the case of real measurements, the results for the Gauss elimination- and Gauss-Jordan method are different from the others. In theory, the difference between these methods is: If, using elementary row operations, the augmented matrix is reduced to row echelon form (REF), then the process is called Gaussian elimination. If the matrix is reduced to reduced row echelon form (RREF), the process is called Gauss-Jordan elimination. For these methods, a large part of the error is of the same order as the input values.

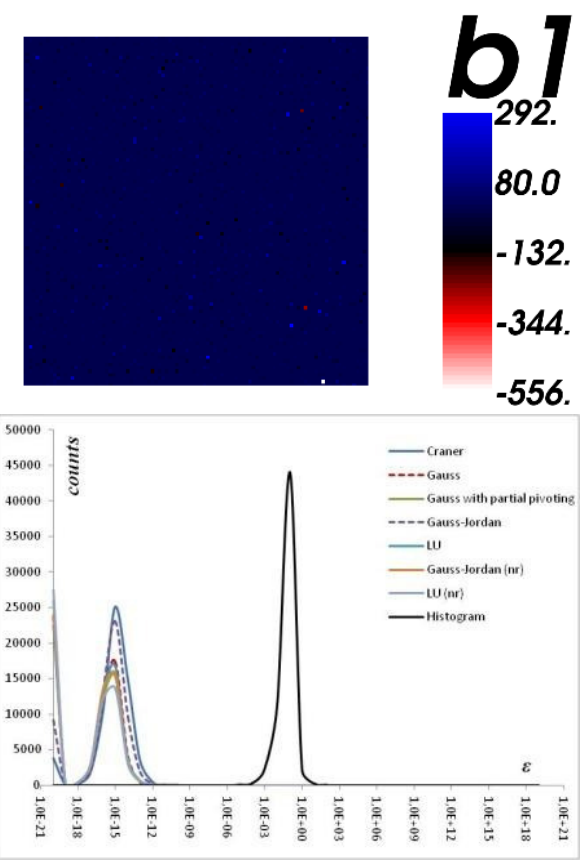



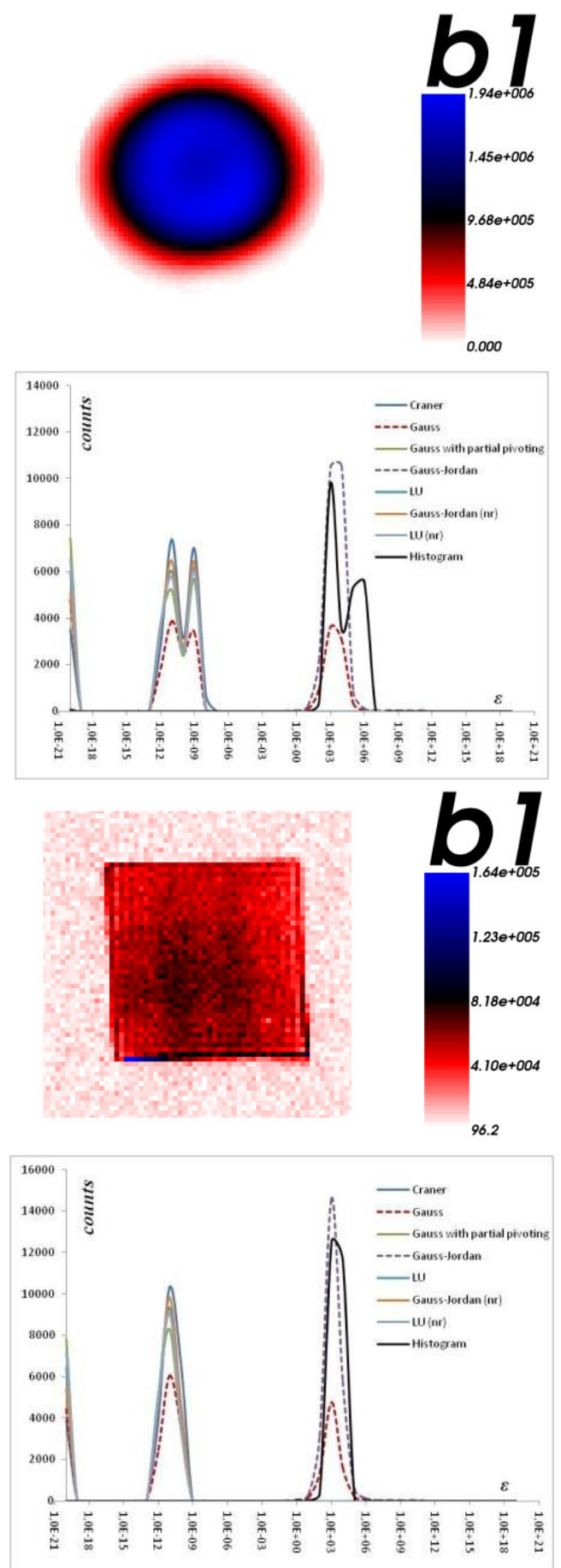

Fig. 2 Distribution of the indirect numerical error for various methods a) random data, b) isotropic phantom, c) anisotropic phantom.

Subsequently the difference between the results obtained with LU method (numerical recipes) and the results obtained in the remaining methods and (Fig.3) were compared. On this basis it can be concluded that the biggest numerical error appears in the noise area. In the case of Gauss and GaussJordan those errors are significantly larger than in the phantom region. In the other methods the errors in both phantom and noise regions are of the same order as the errors in the noise area in Gauss and Gauss-Jordan methods.

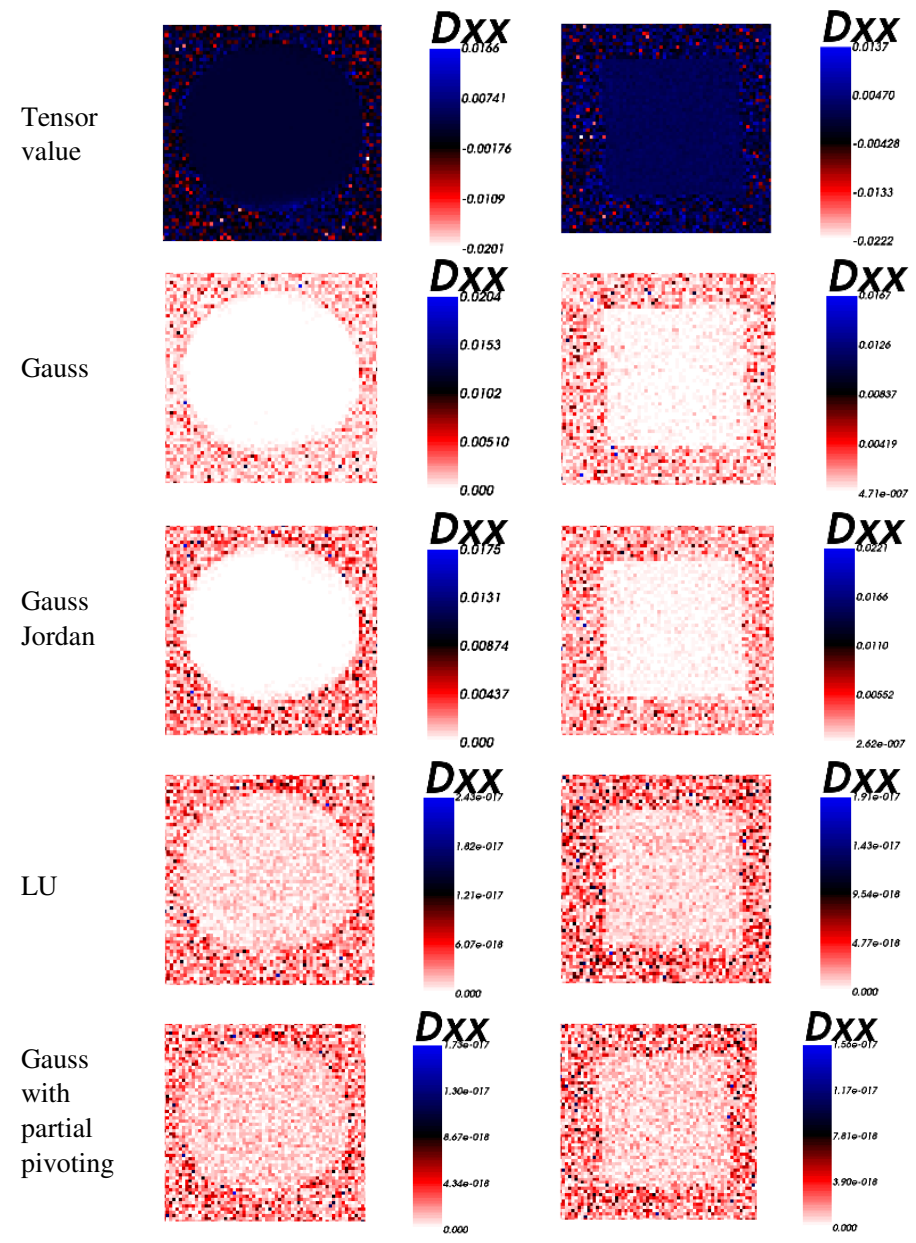

Fig. 3 Distribution of the indirect numerical error for various methods

The last examined aspect was the duration of the calculations. For this purpose, the averaged computing time was determined by repeating the calculations 1000 times for anisotropic phantom (64)64 image) for each of the implemented methods. The fastest method was the Gauss elimination method, the rest were $15-70 \%$ slower. From the previous tests it is known that Gauss method is very prone to numerical errors. The fastest method out of ones giving correct results was the LU, which was $15 \%$ slower than Gauss. if one takes into account the total duration of computing for DTI, i.e. the memory allocation, calculation of own values etc., then the percent difference between Gauss and LU decreases to $8 \%$.

TABLE I.

DEFINING CHARACTERISTICS OF FIVE EARLY DIGITAL COMPUTERS

\begin{tabular}{|l|c|c|c|c|}
\hline & \multicolumn{2}{|c|}{$\begin{array}{c}\text { Time of computing } \\
\text { tensor D }\end{array}$} & \multicolumn{2}{c|}{$\begin{array}{c}\text { Time of computing } \\
\text { BSD }\end{array}$} \\
\hline \multicolumn{1}{|c|}{ Method } & Time [ms] & $\begin{array}{c}\text { Ratio to } \\
\text { Gauss[\%] }\end{array}$ & Time [ms] & $\begin{array}{c}\text { Ratio to } \\
\text { Gauss[\%] }\end{array}$ \\
\hline Gauss & 2.05 & 100.00 & 5.49 & 100.00 \\
\hline Gauss-Jordan & 2.88 & 140.48 & 6.38 & 116.21 \\
\hline LU & 2.36 & 115.12 & 5.95 & 108.37 \\
\hline $\begin{array}{l}\text { Gauss with } \\
\text { partial pivoting }\end{array}$ & 3.49 & 170.24 & 7.04 & 128.23 \\
\hline LU (nr) & 2.54 & 123.90 & 5.92 & 107.83 \\
\hline Gauss-Jordan(nr) & 3.33 & 162.43 & 6.72 & 122.40 \\
\hline
\end{tabular}




\section{CONCLUSION}

Calculating the diffusion tensor D in DTI one has to take into account the impact of the numerical errors, occurring especially in the noise area. Computing time, if all aspects such as the time taken for the allocation of the memory and determining own values and vectors are taken into account, is of minor importance. The methods less affected by the numerical errors, differs in the execution time about $10 \%$. The optimized algorithms will be implement in the B-matrix Spatial Distribution DTI(BSD-DTI) method using a spatial distribution of b-matrix.

\section{ACKNOWLEDGMENT}

The National Centre for Research and Development for grant PBS2/A2/16/2013.

\section{REFERENCES}

[1] Basser PJ, Mattiello J, LeBihan D. MR diffusion tensor spectroscopy and imaging. Biophysical Journal. 1994; 66(1):259-267. W.-K. Chen, Linear Networks and Systems (Book style). Belmont, CA: Wadsworth, 1993, pp. $123-135$.

[2] Krzyżak A. T., et al., Quantitative assessment of Injury in Rats Spinal Cord in vivo using MRI of Water Diffusion Tensor, App. Magnetic Resonance, 2008; 34; 3-20.

[3] Trivedi R., Rathore R. K., Gupta R. K. Review: Clinical application of diffusion tensor imaging. The Indian Journal of Radiology \& Imaging. 2008;18(1):45-52. doi:10.4103/0971-3026.38505.

[4] Mandl R. C. W., Schnack H. G., Zwiers M. P., van der Schaaf A., Kahn R. S., et al. (2008) Functional Diffusion Tensor Imaging: Measuring Task-Related Fractional Anisotropy Changes in the Human Brain along White Matter Tracts. PLoS ONE 3(11): e3631.doi:10.1371/ journal.pone.0003631.

[5] Basser P. J., Mattiello J., LeBihan D. Estimation of the effective selfdiffusion tensor from the NMR spin echo. J. Magn. Reson. B 1994; 103:247-54

[6] Krzyżak A. T., Olejniczak Z., Improving the accuracy of PGSE DTI experiment using spatial distribution of $b$ matrix, Magnetic Resonance Imaging, 2015; 33; 286-295. 